\title{
RESEARCH
}

\section{Glial cells in familial amyloidotic polyneuropathy}

\author{
Nádia Pereira Gonçalves ${ }^{1,2}$, Susete Costelha ${ }^{1}$ and Maria João Saraiva ${ }^{1,2^{*}}$
}

\begin{abstract}
Introduction: Transthyretin V30M mutation is the most common variant leading to Familial Amyloidotic Polyneuropathy. In this genetic disorder, Transthyretin accumulates preferentially in the extracellular matrix of peripheral and autonomic nervous systems leading to cell death and dysfunction. Thus, knowledge regarding important biological systems for Transthyretin clearance might unravel novel insights into Familial Amyloidotic Polyneuropathy pathophysiology. Herein, our aim was to evaluate the ability of glial cells from peripheral and autonomic nervous systems in Transthyretin uptake and degradation. We assessed the role of glial cells in Familial Amyloidotic Polyneuropathy pathogenesis with real-time polymerase chain reaction, immunohistochemistry, interference RNA and confocal microscopy.
\end{abstract}

Results: Histological examination revealed that Schwann cells and satellite cells, from an Familial Amyloidotic Polyneuropathy mouse model, internalize and degrade non-fibrillar Transthyretin. Immunohistochemical studies of human nerve biopsies from V30M patients and disease controls showed intracellular Transthyretin immunoreactivity in Schwann cells, corroborating animal data. Additionally, we found Transthyretin expression in colon of this Familial Amyloidotic Polyneuropathy mouse model, probably being synthesized by satellite cells of the myenteric plexus.

Conclusions: Glial cells from the peripheral and autonomic nervous systems are able to internalize Transthyretin. Overall, these findings bring to light the closest relationship between Transthyretin burden and clearance from the nervous system extracellular milieu.

Keywords: Transthyretin, Internalization, Glial cells, Familial amyloidotic polyneuropathy, Peripheral nervous system, Myenteric plexus

\section{Introduction}

Familial Amyloidotic Polyneuropathy (FAP) is a rare but fulminant and life-threatening neurodegenerative disorder. Approximately ten thousand patients are affected worldwide with endemic foci in Portugal, Japan and Sweden [1]. Major neuropathological and neurochemical hallmarks of this autosomal dominant hereditary disease included extracellular accumulation of mutated transthyretin (TTR) aggregates and amyloid fibers, particularly in autonomic and peripheral nervous systems (ANS and PNS, respectively), leading to sensorimotor, motor and autonomic neuropathy [2].

Transthyretin is a tetramer of identical subunits of 127 amino acid residues each [3]. It is primarily synthesized by the liver and the choroid plexus of the brain $[4,5]$ and functions as a protein carrier for thyroxin and retinol

\footnotetext{
* Correspondence: mjsaraiv@ibmc.up.pt

'Molecular Neurobiology, Instituto de Biologia Molecular e Celular - IBMC, Rua do Campo Alegre 823, Porto 4150-180, Portugal

${ }^{2}$ Instituto de Ciências Biomédicas de Abel Salazar (ICBAS), Universidade do Porto, Porto 4050-313, Portugal
}

[6,7]. More than 100 single point TTR mutations have been discovered, being the exchange of a methionine for a valine at position $30(\mathrm{~V} 30 \mathrm{M})$ the most common in FAP [8]. TTR is mainly produced by the liver as a monomer that assembles into a tetramer and is efficiently secreted. This process occurs in most FAP associated mutations, including carriers of the V30M mutation [9]. Particular high amyloidogenic mutations, such as the L12P associated with leptomeningeal amyloidosis, form intracellular aggregates that are transported into liver lysosomes [10] and thus these mutants are poorly secreted. Contrarily to other TTR amyloidoses, L12P cases present liver TTR deposition [11].

The original amyloid cascade hypothesis proposed that circulating TTR dissociation into non-native monomers is a determinating step for misfolding. Thus, monomers with low conformational stability self-assemble forming non-fibrillar oligomers, protofibrils and mature amyloid fibers $[12,13]$ that accumulate in the extracellular matrix of the gastrointestinal tract, skin, heart, kidney and PNS [14]. 
A particular feature of FAP is organ and tissue tropism for TTR deposition. The mechanistic and functional principles that underlie this fact are not fully understood. Hence, converging evidence revealed the importance of a dynamic balance between formation and clearance of extracellular deposited TTR $[15,16]$. Cellular uptake of soluble TTR by hepatocytes, mouse embryonic fibroblasts, yolk sac cells and sensory neurons, was previously demonstrated in vitro [17-19]. More recently, intracellular material has been observed in fibroblasts and macrophages, through analysis of skin biopsies from FAP patients and TTR transgenic mice [20].

Since important target tissues of TTR load belong to the ANS and PNS, the aim of the present study was to investigate TTR localization in tissues and cells of these systems. To perform this work we take advantage of human nerve biopsies and tissues from a well-established FAP mouse model, carrying the human TTR V30M gene.

We analyzed TTR expression in the peripheral nerve of the FAP mouse model using real-time polymerase chain reaction (qPCR) analysis and determined the subcellular localization of TTR in satellite cells from mice dorsal root ganglia (DRG) and Schwann cells (SC). SC from patients and control subjects were also evaluated, through confocal double immunofluorescence. An interference RNA (RNAi) approach was used to study TTR expression/internalization by satellite cells from the myenteric plexus.

\section{Materials and methods Ethics statement}

All mouse protocols followed the European Union Directive (2010/63/EU) and were previously approved by the Institutional and National General Veterinarian Board ethical committees. Human tissue biopsy was performed after informed consent and approval from the Ethics Committee of the Hospital Geral de Santo António (Porto, Portugal), following the declaration of Helsinki.

\section{Human samples}

Archival sural nerve biopsy samples obtained from FAP V30M patients $(n=4)$ and normal disease control subjects $(n=4)$ were previously characterized, after informed consent, for TTR and amyloid deposition, with immunohistochemistry and Congo red staining, respectively. Samples were kindly provided by the Hospital Geral de Santo António (Porto, Portugal). FAP samples analyzed in this study presented TTR amyloid deposition. Disease control patients were near-relatives of FAP patients who ultimately turned out not to have mutations in TTR. This material was obtained as part of the clinical diagnosis and evaluation of polyneuropathy, before the current use of less invasive methods, as previously described [21].

\section{Animals}

Six months transgenic mice for human TTR V30M, in the 129/Sv and endogenous Ttr null background, heterozygous for the heat shock factor-1 (Hsf-1), here designated as Hsf/ V30M [22], were used for the experiments. Although not presenting amyloid fibers in PNS or ANS, non-fibrillar TTR material is widespread in the extracellular milieu of these systems at 6 months of age. Additionally, 6 monthsold Ttr wild-type (WT) and TTR knockout (KO) mice [23], in a $129 / \mathrm{Sv}$ background were used as controls. Animals were housed in a controlled-temperature room, maintained under a $12 \mathrm{~h}$ light/dark cycle, with water and food ad libitum and euthanized with a lethal injection of a premixed solution containing ketamine $(75 \mathrm{mg} / \mathrm{kg})$ and medetomidine $(1 \mathrm{mg} / \mathrm{kg})$.

\section{Liver TTR silencing in vivo with RNAi}

For TTR-silencing studies, TTR or control siRNA (vehicle) were formulated into a lipid nanoparticle delivery system [24]. Five months-old Hsf/V30M mice were injected in the tail vein with human TTR siRNA $(n=6)$, at a concentration of $1 \mathrm{mg} / \mathrm{kg}$. Untreated age-matched controls received vehicle intravenously $(n=6)$. One injection per week was performed during 4 weeks and animals were sacrificed $48 \mathrm{~h}$ after the last injection. Liver and colon were divided and collected to $10 \%$ formalin and frozen at $-80^{\circ} \mathrm{C}$.

\section{Messenger RNA (mRNA) isolation, complementary DNA (CDNA) synthesis and real-time quantitative polymerase chain reaction (qPCR)}

Liver and colon mRNA ( $n=6$ per group Hsf/V30M; $n=$ $5 \mathrm{WT}$ ) was isolated using phenol extraction (Invitrogen, Carlsbad, CA, USA). Sciatic nerve from Hsf/V30M mice was dissected free from surrounding tissue $(n=5)$ and mRNA extraction performed using RNeasy Mini columns (Qiagen, Gaithersburg, MD, USA). cDNA was synthesized with the SuperScript double-stranded cDNA Kit (Invitrogen). The quality of extracted RNA was assessed with Experion RNA StdSens Analysis Kit (Bio-Rad, Hercules, CA, USA); qPCR was performed in duplicates using iQ Syber Green Super Mix (Bio-Rad) and reactions were run on an Bio-Rad iQ5 software.

Primer sequences were designed using Beacon Designer $8^{\mathrm{TM}}$ (Premier Biosoft, Palo Alto, CA, USA) for TTR (Forward: 5'-ATTCTTGGCAGGATGGCTTC-3', Reverse: 5'-CAGAGGACACTTGGATTCACC-3'); Ttr (Forward: 5'AGCCCTTTGCCTCTGGGAAGAC-3', Reverse: 5'-T GCGATGGTGTAGTGGCGATGG-3'); glyceraldehyde 3phosphate dehydrogenase (Gapdh) (Forward: 5'-GCCTTC CGTGTTCCTACC-3', Reverse: 5'-AGAGTGGGAGTTG CTGTTG-3') and $18 S$ (Forward: 5'-AAATCAGTTATG GTTCCTTTGGTC-3, Reverse: 5'-GCTCTAGAATTACC 
ACAGTTATCCAA-3'). Differential expression was determined by the $2^{\wedge}{ }_{-} \Delta \Delta C T$ method.

\section{Immunohistochemistry}

Liver and colon from animals subjected to TTR siRNA treatment and respective controls were excised, post-fixed in $10 \%$ formalin, embedded in paraffin and cut longitudinally at $3 \mu \mathrm{m}$. Colon from WT and KO mice was used as controls $(n=4)$. Histoclear (National Diagnostics, Atlanta, GA, USA) was used to deparaffinate sections that were thereafter hydrated in a descent alcohol series. Endogenous peroxidase activity was inhibited with $3 \%$ hydrogen peroxide in methanol and sections were blocked with $10 \%$ fetal bovine serum and $0.5 \%$ Triton $\mathrm{x}-100$, in phosphate buffer saline. Primary antibodies against human TTR (1:600, rabbit polyclonal, DAKO, Glostrup, Denmark) and mouse Ttr (1:1500, rabbit polyclonal, Q-Biogen llkirch Cedex, France) were used. After incubation with secondary antibody (anti-rabbit IgG, 1:200, Vector, Burlingame, CA, USA), slides were incubated with avidin-biotin-peroxidase complex (ABC Elite, Vector) and visualized using 3.3'-diaminobenzidine as a chromogen.

\section{Immunofluorescent double labeling}

For double immunofluorescence analyses, sciatic nerve, DRG and colon from Hsf/V30M animals were excised and processed as described above. Human sural nerve biopsies were also used. Rabbit polyclonal anti-human TTR (1:100, DAKO), sheep polyclonal anti-human TTR (1:100, Abcam, Cambridge, UK), mouse monoclonal anti-heparan sulfate proteoglycans (1:100, Amsbio, Tokyo, Japan), goat polyclonal anti-Octamer transcription factor 6 (Oct-6, 1:25, Santa Cruz Biotechnology, Santa Cruz, CA, USA), rabbit polyclonal anti-S100 (1:100, DAKO), rabbit polyclonal antiearly endosome antigen 1 (EEA1, 1:100, Sigma-Aldrich, St. Louis, MO, USA) and mouse monoclonal anti-lysosomalassociated membrane protein 1 (Lamp1, 1:75, Abcam) were used as primary antibodies. Secondary antibodies included donkey anti-rabbit Alexa Fluor 488, donkey anti-goat Alexa Fluor 568, donkey anti-sheep Alexa Fluor 488, goat anti-rabbit Alexa Fluor 568, goat anti-mouse Alexa Fluor 568 and 488 (1:1,000 Molecular Probes, Oregan, USA). Slides were mounted with Vectashield containing 4'.6diamino-2-phenylindole (DAPI) (Vector) and visualized in a laser scanning Confocal Microscope Leica TCS SP5 II (Leica Microsystems, Heidelberg, Germany).
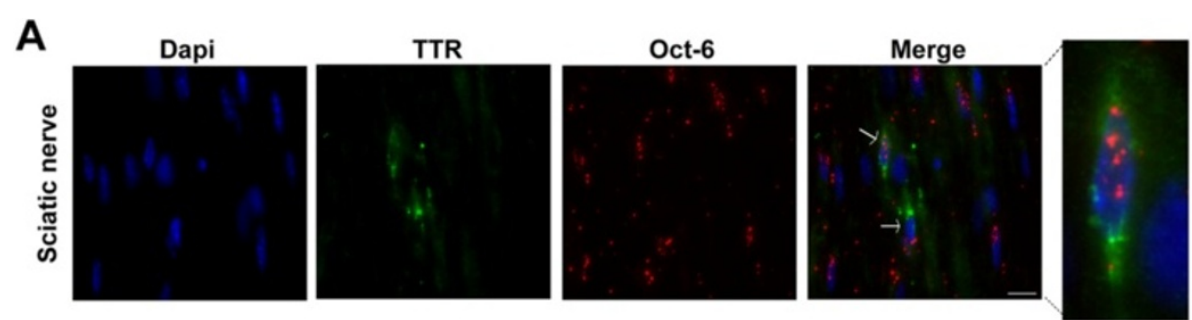

B

Dapi

TTR
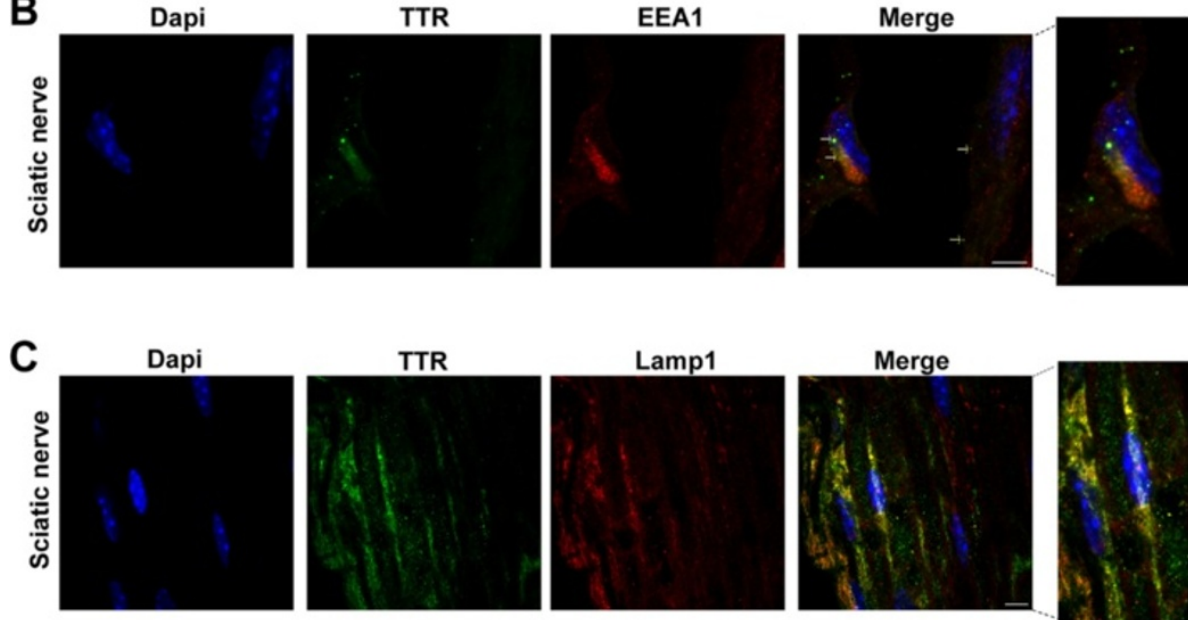

Figure $1 \mathrm{SC}$ express and internalize TTR in the Hsf/V30M FAP mouse model. A) Representative picture of double immunofluorescence between TTR (green) and Oct-6 (red) in HsfN30M nerves, demonstrating TTR intracellular staining in SC (arrows). Superposition of the labels, with DAPI (blue), is shown (merge, $n=4)$. Scale bar $10 \mu \mathrm{m}$. B) Double immunofluorescence of TTR (green) and EEA1 (red) demonstrating colocalization between the 2 proteins in SC of Hsf/N30M mice (arrows). Scale bar $5 \mu \mathrm{m}$. C) Confocal representative image of a sciatic nerve section stained with anti-TTR antibody (green) and a lysosome marker (Lamp1; red). Colocalization between both markers was noticed in yellow; scale bar $5 \mu \mathrm{m}$. 
TTR-immunopositive SC in human sural nerve biopsies were detected by merged images with anti-TTR antibody and a SC marker (Oct-6). The number of TTRimmunopositive $\mathrm{SC}$ in each group was calculated as an average of 5 visual fields $(447.63 \mu \mathrm{m} \times 335.40 \mu \mathrm{m})$ per sample ( $n=4$ per group) at an original magnification of 20x, in a Axio Imager (Zeiss, Hertfordshire, UK).

\section{Statistical analysis}

Two or three groups' comparison was performed with Student T-test or One-way ANOVA, respectively. For Oneway ANOVA, Bonferroni was used as the post test. Data are expressed as mean values \pm standard error of the mean (SEM) and $p$-values of less than 0.05 were considered to be significant $\left(p^{*}<0.05, p^{* *}<0.01\right.$ and $\left.{ }^{* * * *} p<0.001\right)$.

\section{Results}

Non-fibrillar TTR is produced and degraded by Schwann cells in the FAP mouse model Hsf/V30M

Expression of TTR by SC of sciatic nerve in a mouse model carrying the TTR V30M mutation but missing TTR deposition in the PNS and ANS was previously described [25]. Thus, we questioned whether this feature is recapitulated in an FAP mouse model deficient for the Hsf-1 (Hsf/ V30M). In this model, non-fibrillar TTR deposition is widespread along the gastrointestinal tract since the first month of age. Furthermore regarding the nervous system, TTR deposits are found in the extracellular matrix of sciatic nerve, DRG and parasympathetic ganglia approximately at 6 months of age [22]. For this reason, animals with 6 months-old were chosen for subsequent analyses.

Using qPCR, TTR expression by the sciatic nerve of Hsf/V30M mice was found (61.7 units ratio of TTR mRNA and Gapdh mRNA \pm 16.2 SEM). With double immunofluorescence between TTR and Oct-6, a SC marker, TTR intracellular staining in these cells was noticed (Figure 1A, arrows), suggesting them as the primary source of TTR in nerve, in accordance with previous results from other FAP mouse models [25,26].

To localize TTR within SC, additional studies were performed using double immunofluorescence between TTR and a marker for early endosome antigen 1 (EEA1). Looking for cells previously stained with Oct-6, consecutive cross-sectional images revealed that indeed SC presented intracellular punctuate material, visible in higher magnification, that colocalize with EEA1 (Figure 1B, arrows). Further labeling with TTR and Lamp1 demonstrated colocalization of V30M TTR with lysosomes (Figure 1C), indicating that $\mathrm{SC}$, might also be important for TTR clearance.
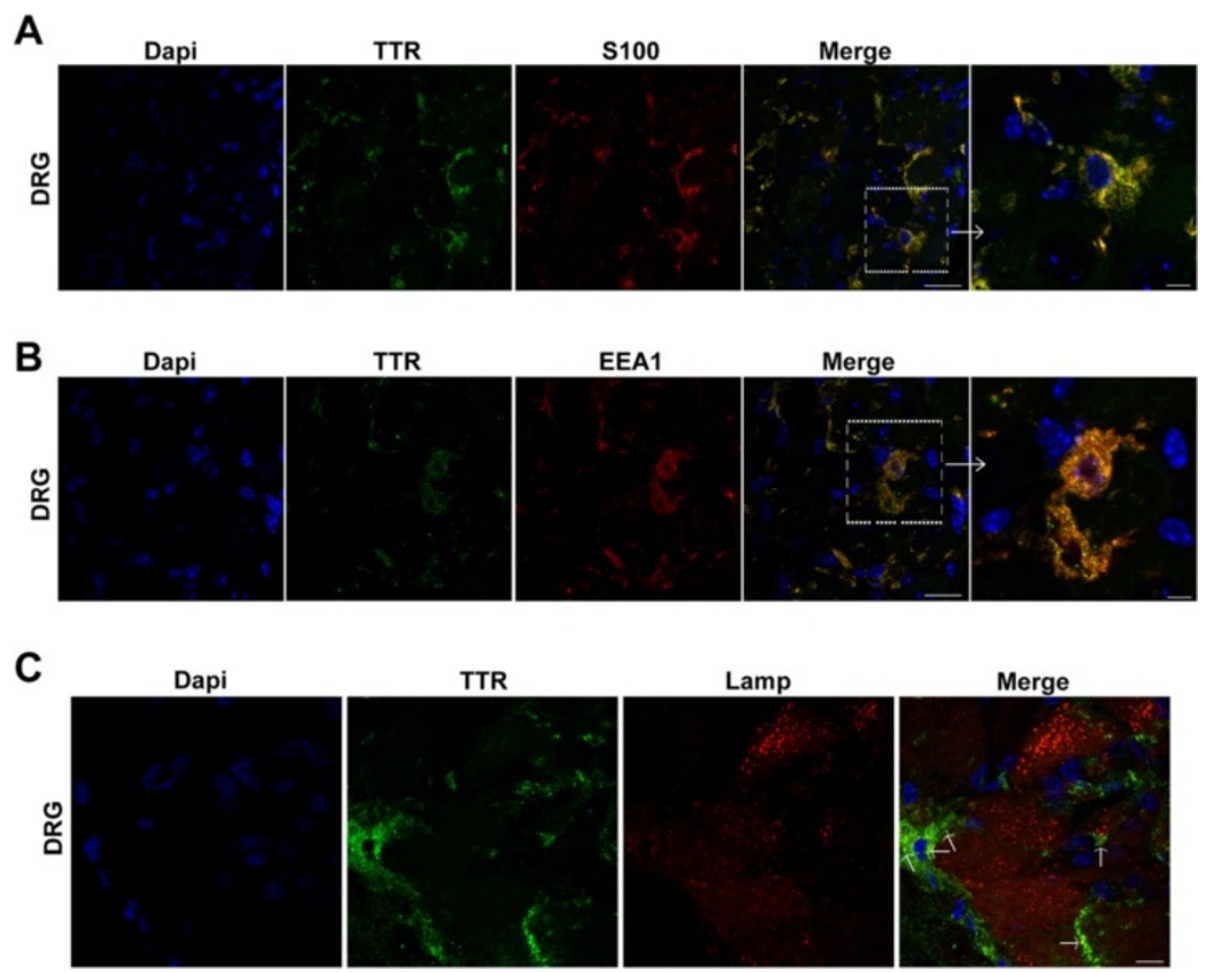

Figure 2 Glial cells from DRG internalize TTR, in a mouse model of FAP. A) Double immunofluorescence of TTR (green) and S100 (red), a marker for glial cells, in DRG of Hsf/N30M mice, showing TTR localized inside satellite cells. Scale bar $20 \mu \mathrm{m}$; higher magnification $5 \mu \mathrm{m}$. B) TTR colocalizing with EEA1 in satellite cells from DRG of Hsf/N30M animals. Scale bar $20 \mu \mathrm{m}$; higher magnification $5 \mu \mathrm{m}$. C) Representative confocal image demonstrating colocalization between TTR (green) and lysosomes (Lamp1; red) in satellite cells from DRG (arrows). Scale bar 8 m. 
Non-fibrillar TTR is internalized by glial cells of sensory ganglia

Data reported so far indicate that $\mathrm{SC}$, besides being a source of TTR might also be important for TTR clearance in the sciatic nerve. We next investigated TTR immunoreactive glial cells from DRG of Hsf/V30M mice. In fact, with double immunofluorescence between TTR and S100, TTR intracellular staining was found in glial cells of DRG (possible satellite cells; Figure 2A). Since it was previously shown that TTR is not synthesized by cells of DRG [27] and in this work we found colocalization of TTR with EEA1 (Figure 2B), we hypothesized that satellite cells have an active role in TTR internalization. Moreover, we found TTR signal partially colocalized with lysosomes (Figure 2C); therefore we can conclude that these cells participate in TTR clearance in this animal model.

Intracellular TTR in human Schwann cells

It was previously found that TTR fibril extracts contain heparan sulfate proteoglycans [28,29]. Thus, we started by characterizing TTR deposits on sural nerve biopsies from FAP patients regarding this glycosaminoglycan. As illustrated in merged panel of Figure 3A, heparan sulfate colocalized with TTR deposits, demonstrating that heparan sulfate is present in TTR deposits as co-localized molecules. Following the previous results with the FAP mouse model, we wondered if intracellular TTR could also be observed in nerves from FAP patients. To address this
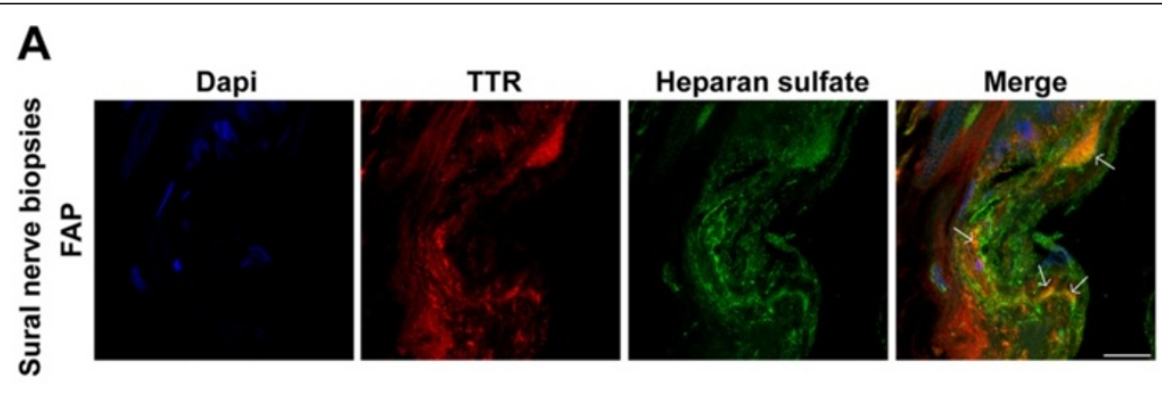

B

Dapi
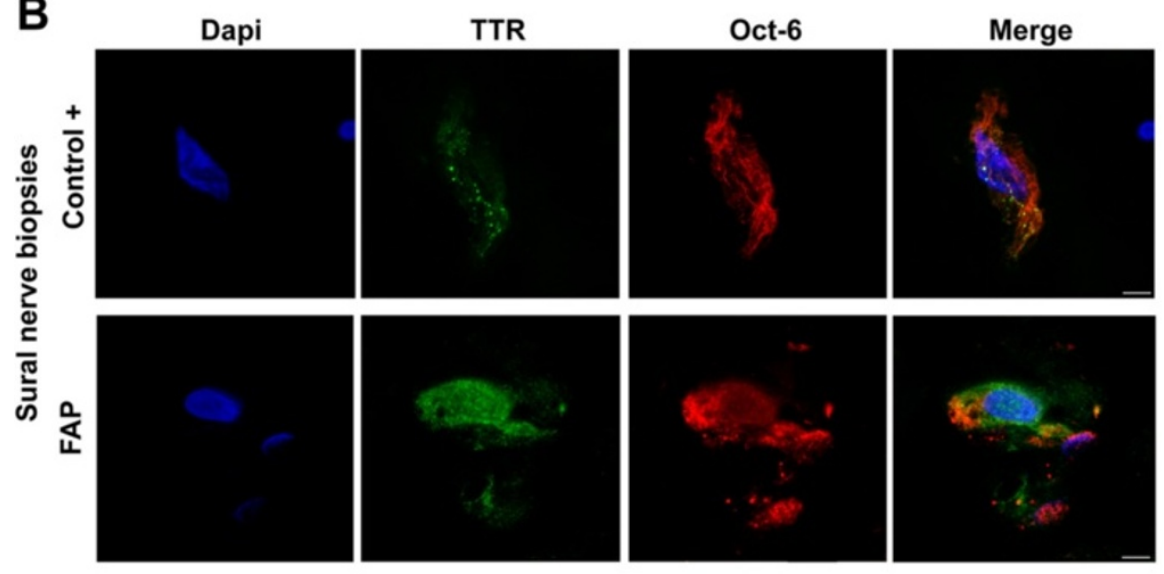

C
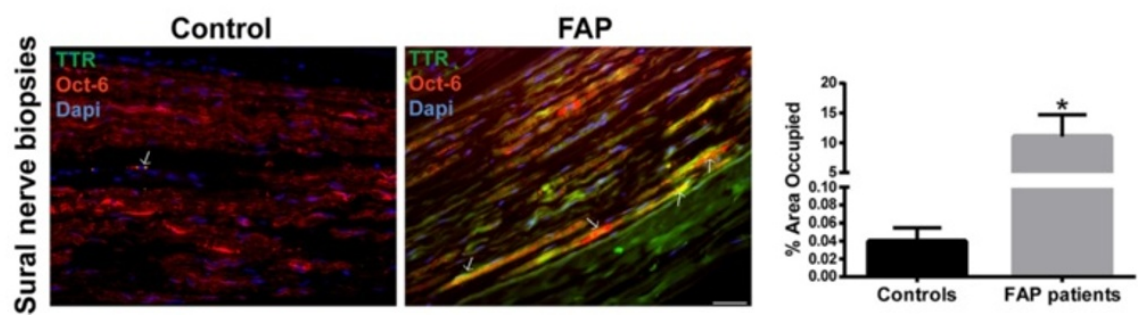

Figure 3 Intracellular TTR in SC of human nerve biopsies. A) Representative photomicrographs obtained with confocal microscopy showing colocalization (yellow and orange in merged panel) between TTR (red) and heparan sulfate (green) in sural nerve biopsies from FAP patients. Scale bar $15 \mu \mathrm{m}$. B) Representative images from double immunofluorescence between TTR (green) and Oct-6 (red) in sural nerve biopsies from disease control individuals and FAP patients, demonstrating TTR intracellular staining in SC. Superposition of the labels, with DAPI (blue), is shown (merge, $n=4)$. Scale bar $4 \mu \mathrm{m}$. C) Low magnification images from longitudinal sections of human sural nerves showing colocalization between TTR and Oct-6 (arrows); scale bar $40 \mu \mathrm{m}$. Chart represents quantification of TTR positive SC, related to total SC in nerve sections. Results are presented as means \pm SEM $\left(p^{*}<0.05\right)$. 
issue, we also used sural nerve biopsies from normal individuals (disease free) as controls. In disease control individuals, the majority of SC were negative for WT TTR intracellular staining; surprisingly, few positive cells presented WT TTR immunoreactive punctuate material (Figure 3B, top panel, and $3 \mathrm{C}$ ), suggesting that SC might have an important role for uptake and degradation of WT TTR reaching the nerve though the blood stream. In FAP carriers, some SC had generalized V30M TTR intracellular staining (Figure 3B, bottom panel, and 3C) and the percentage of TTR positive SC was significantly higher than for disease control patients (Figure 3C). TTR colocalization with EEA1 was observed in both cases (Figure 4A, arrows), corroborating the notion that punctuate intracellular material arise from TTR internalization and not from cell synthesis. Colocalization between TTR and lysosomes was scarce (Figure 4B, arrow), most likely related to sample storage and processing.

Intracellular TTR in satellite cells from the myenteric plexus In FAP disease, ANS is severely affected by TTR deposition ultimately leading to bladder dysfunction, diarrhea, malabsorption and motility disturbances [14]. Thus, we questioned whether satellite cells from the myenteric plexus may also be able to internalize TTR in FAP. By histological analysis of Hsf/V30M mice colon, non-fibrillar TTR immunostaining was found in Auerbach plexus (myenteric plexus) especially inside smaller cells surrounding the nerve cell bodies (compatible with satellite cells), in contrast with WT and TTR KO animals that were negative for TTR (Figure 5A). Following these results, we performed double immunofluorescence between TTR and S100 in colon sections of Hsf/V30M animals. Colocalization between the two proteins was observed (Figure 5B). Since the TTR staining present in these cells partially colocalized with EEA1 (Figure 5C, arrows) and Lamp1 (Figure 5D, arrows), it is reasonable to suggest that satellite cells have an important role in non-fibrillar TTR uptake and internalization. However, we could not discard the possibility of TTR synthesis by these cells of myenteric plexus, especially when $T T R$ production in colon was already previously suggested [30]. To address this question we treated 5 months-old Hsf/V30M mice with TTR siRNA, to inhibit TTR deposition in the gastrointestinal tract. With this treatment we were able to silence 92\% TTR expression by the liver (Figure 6A and B), avoiding TTR circulation in plasma and burden in tissues, as previously described [31]. Nevertheless, TTR immunostaining in satellite cells from myenteric plexus was equally observed in animals treated with TTR siRNA or controls (vehicle treated mice) (Figure 6C). By qPCR we found TTR expression in colon of Hsf/V30M mice,
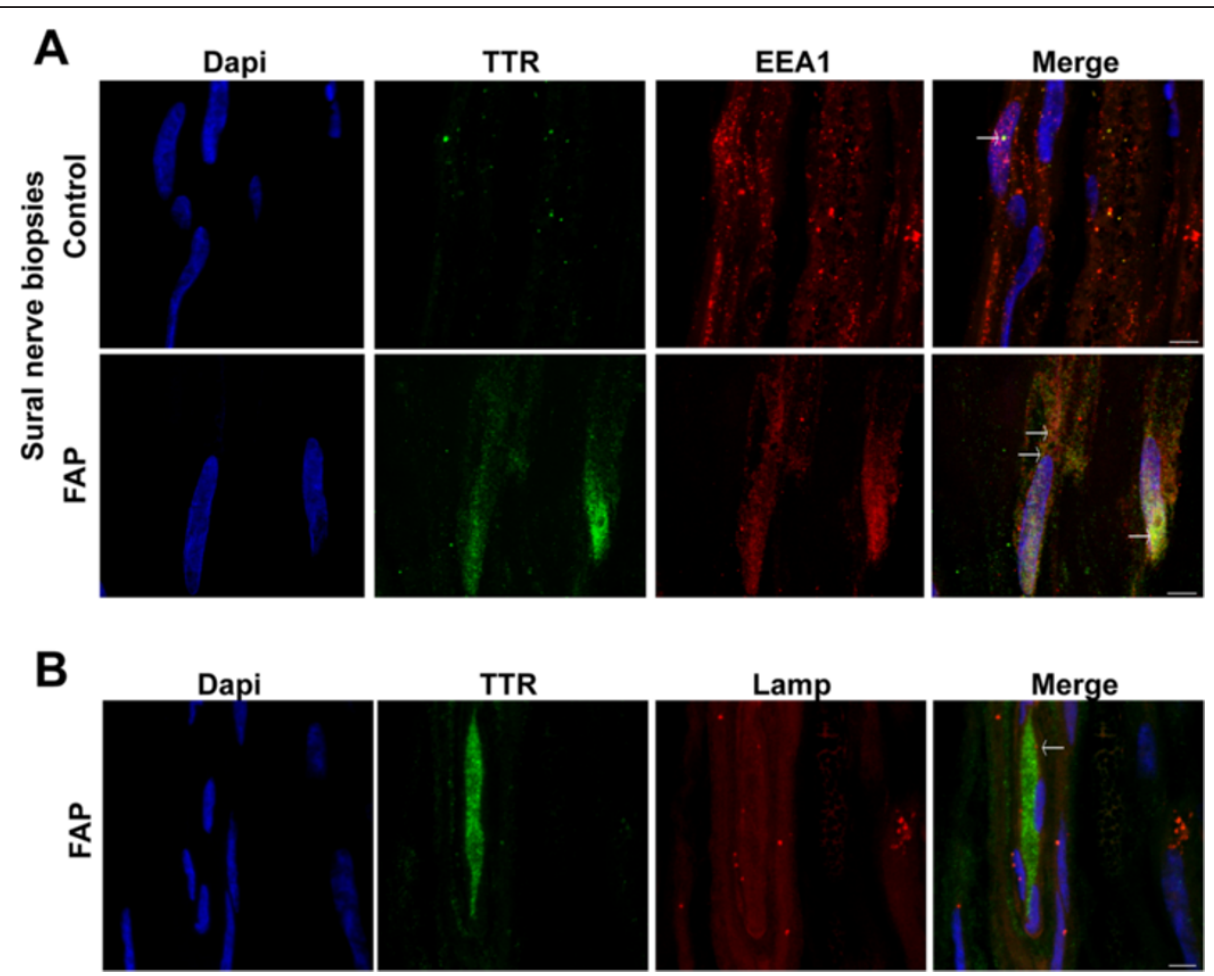

Figure 4 TTR is internalized by human SC. A) Double immunofluorescence of TTR (green) and EEA1 (red) showing colocalization spots between the 2 proteins in SC of FAP patients and normal control individuals (arrows). Scale bar $4 \mu \mathrm{m}$. B) Confocal representative image showing scarce colocalization between TTR (green) and lysosomes (red). Scale bar $3 \mu \mathrm{m}$. 
A

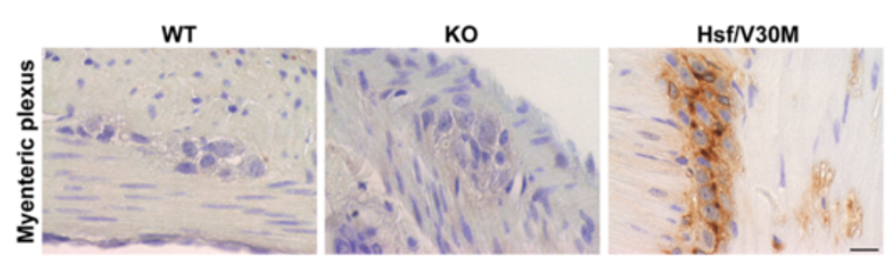

B

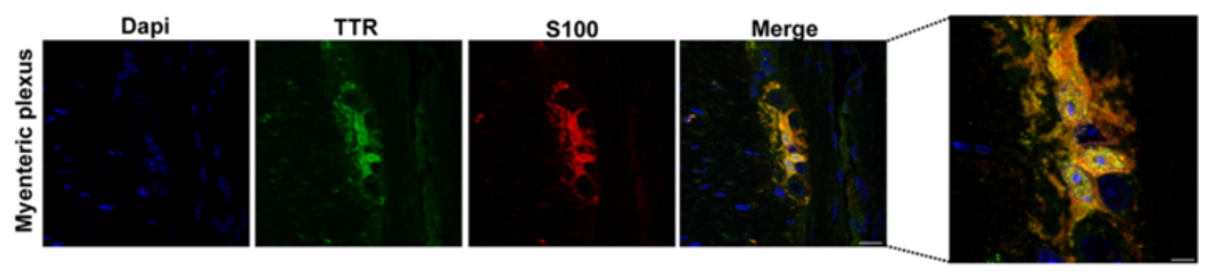

C
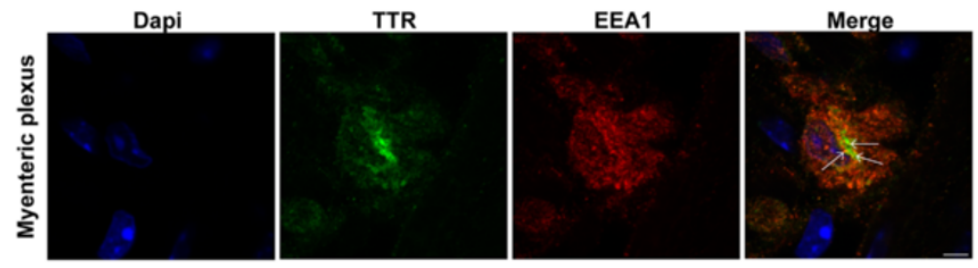

D
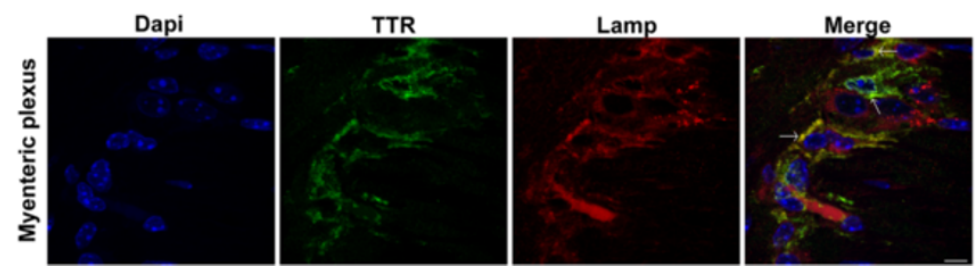

Figure $\mathbf{5}$ TTR is internalized by satellite cells from the myenteric plexus. A) TTR immunohistochemical staining on colon myenteric plexus from WT, TTR KO and HsfN30M animals. Scale bar $20 \mu \mathrm{m}$. B) Double immunofluorescence between TTR (green) and S100 (red), a satellite cell marker, showing TTR inside these cells. Scale bar $14 \mu \mathrm{m}$; higher magnification $5 \mu \mathrm{m}$. C) Representative confocal image of myenteric plexus section showing a satellite cell with intracellular TTR staining (green) colocalizing with EEA1 (red) (arrows). Scale bar 4 um. D) Confocal microscopy image with anti-TTR antibody (green) and lysosome marker (Lamp1; red). Nuclei stained blue with DAPI. Arrows indicate colocalization of TTR with Iysosomes. Scale bar $5 \mu \mathrm{m}$.

with similar levels of relative expression between siRNA and vehicle treated mice (Figure 6D). Additionally, Ttr expression in colon from WT mice was considerably lower than in Hsf/V30M mice (Figure 6D). This, together with the fact that no TTR reactivity was found in satellite cells from myenteric plexus in WT mice, suggests that FAP colonic myenteric plexus might be able to synthesize and clear TTR.

\section{Discussion}

FAP is a peculiar form of neuropathy with clinical symptoms generally occurring in the second or third decade of life. However, a late-onset form of disease has also been described, affecting individuals over 50 years of age and characterized by low penetrance rate and different pathological presentations [32]. Sensory impairment, wasting and weakness results from neuronal and axonal loss consequent to obliteration/dysfunction of small vessels supplying the nerve tissue, nerve fiber compression by TTR deposits or toxic effects of amyloid precursors [32,33]. Additionally, motor sensory type is often found in FAP, characterized by muscle atrophy with distal predominance and decreased motor conduction velocity [34].

In peripheral nerve, TTR deposits are predominantly found in the endoneurium, in close contact with SC and collagen fibrils [2]. Thus, an impairment of axon-SC interaction due to SC dysfunction might contribute to axonopathy. Similarly, in nervous ganglia, TTR deposition occurs in the stroma in close association to satellite cells [35-37]. Satellite cells covering sensory neurons are similar to the $\mathrm{SC}$ of peripheral nerve as they are both derived from the neural crest of the embryo during development [38]. They are known as glial supporting cells of the nervous system and have important roles on mechanical support to 


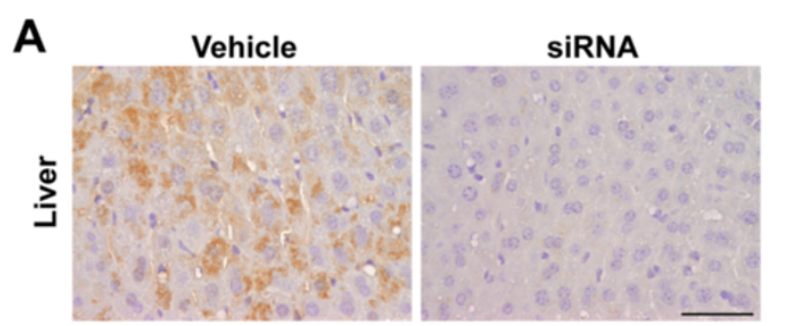

C
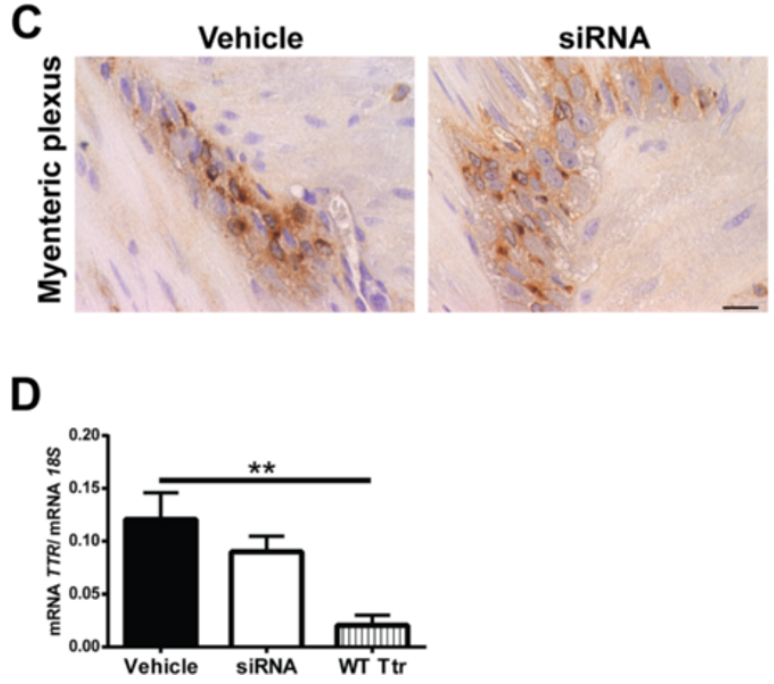

B

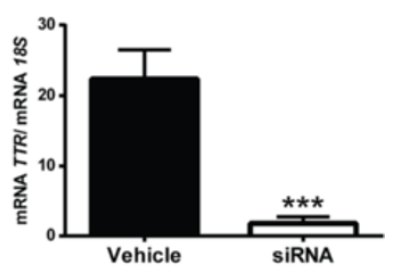

Figure $6 T T R$ is expressed by colon of the Hsf/V30M FAP mouse model. A) Photomicrographs illustrating TTR staining in liver of Hsf/N30M mice, treated with TTR siRNA or vehicle; scale bar $50 \mu \mathrm{m}$. B) Histogram represents TTR mRNA levels in liver of HsfN30M mice treated with TTR siRNA as compared with control animals receiving only vehicle. Data was normalized using 185 as the housekeeping gene ( $\left.p^{* * *}<0.001\right)$. C) Histological images of colonic myenteric plexus from HsfN30M mice treated with TTR siRNA as compared with vehicle treated animals. Scale bar 20 $\mu$ m. D) Histogram denoting TTR mRNA expression in colon from HsfN30M mice treated with vehicle or siRNA and Ttr mRNA from WT animals. Data was normalized using 185 as the housekeeping gene $\left(p^{* *}<0.01\right)$.

neurons, nutrients/oxygen supply and removal of cell debris [39]. TTR expression by glial cells of sensory neurons was previously suggested [40]. However, this hypothesis was later disputed and the results were attributed to TTR contamination by the meninges [27]. Recently, TTR gene expression by SC of peripheral nerve was reported [25]. Accordingly, in the present study, we also observed TTR expression in the peripheral nerve of Hsf/V30M mice, corroborating the previous data.

Glial cells regulate neurons microenvironment and appear to be actively engaged in the control of extracellular matrix composition [39]. Thus, several studies indicate the ability of these cells in the uptake of different substances [41-45]. Since TTR non-fibrillar deposits are found in the extracellular matrix in close association with glial cells, we decided to investigate whether these cells in sensory neurons and peripheral nerve might be important for TTR uptake in vivo. In fact we found immunoreactive TTR in satellite and SC, both in the Hsf/V30M transgenic model and in tissues from FAP patients. Occasionally, the observed intracellular TTR presented a punctate-like pattern, compatible with its presence within vesicles. Once in the cell, endocytic vesicles are rapidly targeted to the early endosomes. Thus, despite the existence of numerous internalization routes, early endosomes are a focal point of the endocytic pathway $[46,47]$. EEA1 is a widely used marker for early endosomes due to its colocalization with the transferrin receptor [48]. In this work, TTR colocalization with EEA1 and a lysosome marker (Lamp1) in glial cells of peripheral nerve and sensory ganglia indicate internalization and consequent vesicular transport of TTR through early endosomes for degradation.

In Alzheimer's disease, $\mathrm{A} \beta$ internalization by glia has been observed in vitro and in vivo $[43,49]$ suggesting an important role for these cells in Alzheimer pathology. Nevertheless, exposure of glial cells to A $\beta$ aggregates could have detrimental consequences with upregulation of inflammatory mediators and nitric oxide, resulting in glia and neuron cell death [50].

Another striking feature of FAP is severe autonomic nervous dysfunction, affecting particularly the gastrointestinal tract [14,32]. Initial gastrointestinal symptoms are severe constipation alternating with periods of diarrhea, nausea and vomiting [51]. Evidence suggests that 
environmental and genetic factors have impact on the clinical pattern of FAP [52]. For instance, in Japanese FAP patients no significant destruction of the enteric nervous system is observed [53] while Portuguese FAP patients present infiltration of amyloid material in the space between two adjacent ganglia, accompanied by different degrees of neuronal loss [54]. However, the mechanisms leading to neuron cell death remain poorly understood. Some factors that have been associated with the pathogenesis of gastrointestinal dysfunction are a depletion of neuroendocrine cells, such as serotonin, somatostatin or PYY immunoreactive cells [55], accumulation of advanced glycation end products [56], loss of interstitial cells of Cajal [57] and amyloid deposition in sympathetic ganglia [58]. Although amyloid deposits have not been found in the myenteric plexus of Hsf/V30M FAP mouse model, this was the first animal model presenting nonfibrillar TTR deposition in the autonomic nervous system of the GI tract [22]. Therefore, we next investigated whether satellite cells were able to internalize TTR, also in this system. TTR colocalization with EEA1 and lysosomes indicated that endocytic trafficking pathways are activated in satellite cells from the myenteric plexus of Hsf/V30M mice. Importantly, this is the first study showing TTR synthesis by the enteric tissue, in this FAP mouse model. Therefore, it is reasonable to suggest that satellite cells may also be synthesizing mutated TTR which in turn might contribute for the non-fibrillar TTR deposition. It would be interesting to confirm these results in biopsy colon specimens from FAP patients, however such specimens are very difficult to obtain since the conditions of these patients not often calls for colonoscopy.

Overall, the present study brings to light new insights into the FAP pathophysiology. Besides TTR endocytosis by fibroblasts demonstrated both in vitro and in vivo [20], TTR internalization in vivo by glial cells of peripheral nerve, sensory ganglia and myenteric plexus was here demonstrated. However, whether TTR uptake by these cells is neuroprotective or neurotoxic leading to glial and neuronal cell death with autonomic dysfunction needs further investigation. Furthermore, additional studies are needed to clarify the molecular mechanisms and signaling platforms involved in these particular systems.

\section{Conclusions}

The results presented in the current study confirm previous findings that TTR is expressed on peripheral nerve and colon, possibly by glial cells. SC and satellite cells from sciatic nerve, DRG and myenteric plexus are able to internalize and degrade TTR in the Hsf/V30M mouse model, contributing for TTR extracellular clearance. Additionally, TTR colocalization with EEA1 was found in SC from human patients, suggesting a dual role for these cells in FAP. Therefore, an imbalance of this system might trigger or accelerate TTR aggregates deposition in target tissues. These novel data regarding the physiopathology of FAP might open new windows of action in the design of new therapeutic targets.

\section{Abbreviations}

ANS: Autonomic nervous system; DRG: Dorsal root ganglia; EEA1: Early endosome antigen-1; FAP: Familial amyloidotic polyneuropathy; Gapdh: Glyceraldehyde 3-phosphate dehydrogenase; Hsf-1: Heat shock factor 1; KO: Knockout; Lamp1: Lysosomal-associated membrane protein 1; PNS: Peripheral nervous system; qPCR: Real-time polymerase chain reaction; SC: Schwann cells; SEM: Standard error of the mean; TTR: Transthyretin; WT: Wild-type; RNAi: Interference RNA.

\section{Competing interests}

The authors declare that they have no competing interests.

\section{Authors' contributions}

NPG performed confocal microscopy, immunohistochemistry, treatment with RNAi and statistical analysis. SC carried out the GPCR. MJS provided reagents and helped for interpretation of data. NPG and MJS performed study design and wrote the manuscript. All authors read and approved the final manuscript.

\section{Acknowledgements}

We would like to thank Paula Gonçalves from IBMC for paraffin tissue processing and Alnylam Pharmaceuticals (Boston) for the kind supply of siRNA reagents. This work was funded by FEDER funds through the Operational Competitiveness Program - COMPETE and by National Funds through FCT - Fundação para a Ciência e a Tecnologia under the project FCOMP-01-0124-FEDER-028406 (PTDC/BIM-MEC/0282/2012) and fellowship to NPG (SFRH/BD/74304/2010). The present work was sponsored by POPH/FSE QREN program and also financed by a grant from Cordex.

Received: 9 October 2014 Accepted: 8 December 2014 Published online: 18 December 2014

\section{References}

1. Said G, Grippon S, Kirkpatrick P (2012) Tafamidis. Nat Rev Drug Discov 11:185-186

2. Coimbra A, Andrade C (1971) Familial amyloid polyneuropathy: an electron microscope study of peripheral nerve in five cases. II. nerve fibril changes. Brain 94:207-212

3. Blake CC, Geisow MJ, Oatley SJ, Rérat B, Rérat C (1978) Structure of prealbumin: secondary, tertiary and quaternary interactions determined by Fourier refinement at 1.8. J Mol Biol 121:339-356

4. Aleshire SL, Bradley CA, Richardson LD, Parl FF (1983) Localization of human prealbumin in choroid plexus epithelium. J Histochem Cytochem 31:608-612

5. Soprano DR, Herbert J, Soprano KJ, Schon EA, Goodman DS (1985) Demonstration of transthyretin mRNA in the brain and other extrahepatic tissues in the rat. J Biol Chem 260:11793-11798

6. Kanai M, Raz A, Goodman DS (1968) Retinol-binding protein: the transport protein for vitamin A in human plasma. J Clin Invest 47:2025-2044

7. Woeber KA, Ingbar SH (1968) The contribution of thyroxine-binding prealbumin to the binding of thyroxine in human serum, as assessed by immunoadsorption. J Clin Invest 47:1710-1721

8. Saraiva MJ, Birken S, Costa PP, Goodman DS (1984) Amyloid fibril protein in familial amyloidotic polyneuropathy, Portuguese type. definition of molecular abnormality in transthyretin (prealbumin). J Clin Invest 74:104-119

9. Sekijima Y, Wiseman RL, Matteson J, Hammarström P, Miller SR, Sawkar AR, Balch WE, Kelly JW (2005) The biological and chemical basis for tissue-selective amyloid disease. Cell 121:73-85

10. Batista AR, Sena-Esteves M, Saraiva MJ (2013) Hepatic production of transthyretin L12P leads to intracellular lysosomal aggregates in a new somatic transgenic mouse model. Biochim Biophys Acta 1832:1183-1193

11. Brett M, Persey MR, Reilly MM, Revesz T, Booth DR, Booth SE, Hawkins PN, Pepys MB, Morgan-Hughes JA (1999) Transthyretin Leu12Pro is associated with systemic, neuropathic and leptomeningeal amyloidosis. Brain 122:183-190 
12. McCutchen SL, Lai Z, Miroy GJ, Kelly JW, Colón W (1995) Comparison of lethal and nonlethal transthyretin variants and their relationship to amyloid disease. Biochemistry 34:13527-13536

13. Quintas A, Vaz DC, Cardoso I, Saraiva MJ, Brito RM (2001) Tetramer dissociation and monomer partial unfolding precedes protofibril formation in amyloidogenic transthyretin variants. J Biol Chem 276:27207-27213

14. Sousa MM, Saraiva MJ (2003) Neurodegeneration in familial amyloid polyneuropathy: from pathology to molecular signaling. Prog Neurobiol 71:385-400

15. Tsuchiya A, Yazaki M, Kametani F, Takei Y, Ikeda S (2008) Marked regression of abdominal fat amyloid in patients with familial amyloid polyneuropathy during long-term follow-up after liver transplantation. Liver Transpl 14:563-570

16. Tsuchiya-Suzuki A, Yazaki M, Kametani F, Sekijima Y, Ikeda S (2011) Wild-type transthyretin significantly contributes to the formation of amyloid fibrils in familial amyloid polyneuropathy patients with amyloidogenic transthyretin Val30Met. Hum Pathol 42:236-243

17. Sousa MM, Norden AG, Jacobsen C, Willnow TE, Christensen El, Thakker RV, Verroust PJ, Moestrup SK, Saraiva MJ (2000) Evidence for the role of megalin in renal uptake of transthyretin. J Biol Chem 275:38176-38181

18. Sousa MM, Saraiva MJ (2001) Internalization of transthyretin. evidence of a novel yet unidentified receptor-associated protein (RAP)-sensitive receptor. J Biol Chem 276:14420-14425

19. Fleming CE, Mar FM, Franquinho F, Saraiva MJ, Sousa MM (2009) Transthyretin internalization by sensory neurons is megalin mediated and necessary for its neuritogenic activity. J Neurosci 29:3220-3232

20. Misumi Y, Ando Y, Gonçalves NP, Saraiva MJ (2013) Fibroblasts endocytose and degrade transthyretin aggregates in transthyretin related amyloidosis. Lab Invest 93:911-920

21. Sousa MM, Du Yan S, Fernandes R, Guimaraes A, Stern D, Saraiva MJ (2001) Familial amyloid polyneuropathy: receptor for advanced glycation end products-dependent triggering of neuronal inflammatory and apoptotic pathways. J Neurosci 21:7576-7586

22. Santos SD, Fernandes R, Saraiva MJ (2010) The heat shock response modulates transthyretin deposition in the peripheral and autonomic nervous systems. Neurobiol Aging 31:280-289

23. Episkopou V, Maeda S, Nishiguchi S, Shimada K, Gaitanaris GA, Gottesman ME, Robertson EJ (1993) Disruption of the transthyretin gene results in mice with depressed levels of plasma retinol and thyroid hormone. Proc Natl Acad Sci U S A 90:2375-2379

24. Semple SC, Akinc A, Chen J, Sandhu AP, Mui BL, Cho CK, Sah DW, Stebbing D, Crosley EJ, Yaworski E, Hafez IM, Dorkin JR, Qin J, Lam K, Rajeev KG, Wong KF, Jeffs LB, Nechev L, Eisenhardt ML, Jayaraman M, Kazem M, Maier MA, Srinivasulu M, Weinstein MJ, Chen Q, Alvarez R, Barros SA, De S, Klimuk SK, Borland T et al (2010) Rational design of cationic lipids for siRNA delivery. Nat Biotechnol 28:172-176

25. Murakami T, Ohsawa Y, Zhenghua L, Yamamura K, Sunada Y (2010) The transthyretin gene is expressed in Schwann cells of peripheral nerves. Brain Res 1348:222-225

26. Gonçalves NP, Teixeira-Coelho M, Saraiva MJ (2014) The inflammatory response to sciatic nerve injury in a Familial Amyloidotic Polyneuropathy mouse model. Exp Neurol 257:76-87

27. Sousa MM, Saraiva MJ (2008) Transthyretin is not expressed by dorsal root ganglia cells. Exp Neurol 214:362-365

28. Noborn F, O'Callaghan P, Hermansson E, Zhang X, Ancsin JB, Damas AM, Dacklin I, Presto J, Johansson J, Saraiva MJ, Lundgren E, Kisilevsky R, Westermark P, Li JP (2011) Heparan sulfate/heparin promotes transthyretin fibrillization through selective binding to a basic motif in the protein. Proc Natl Acad Sci U S A 108:5584-5589

29. Bourgault S, Solomon JP, Reixach N, Kelly JW (2011) Sulfated glycosaminoglycans accelerate transthyretin amyloidogenesis by quaternary structural conversion. Biochemistry 50:1001-1015

30. Loughna S, Bennett P, Moore G (1995) Molecular analysis of the expression of transthyretin in intestine and liver from trisomy 18 fetuses. Hum Genet 95:89-95

31. Alvarez R, Borland T, Chen Q, Milstein S, Nguyen T, Hinkle G, Kuchimanchi S, Costigan J, Ristoiu V, Wang G, Cole G, Dorkin R, Akinc A, Nechev L, Kosovrasti V, Tchangov L, Tracy M, Jeffs L, MacLachlan I, Lutwyche P, Martins D, Costelha S, Saraiva MJ, Sah DWY (2010) ALN-TTR, an RNAi therapeutic for the treatment of transthyretin-mediated Amyloidosis. In: Abstracts of the XIlth International Symposium on Amyloidosis, Rome, Italy, 18-21 April 2010
32. Koike H, Misu K, Sugiura M, lijima M, Mori K, Yamamoto M, Hattori N, Mukai E, Ando Y, Ikeda S, Sobue G (2004) Pathology of early- vs late-onset TTR Met30 familial amyloid polyneuropathy. Neurology 63:129-138

33. Sousa MM, Cardoso I, Fernandes R, Guimarães A, Saraiva MJ (2001) Deposition of transthyretin in early stages of familial amyloidotic polyneuropathy: evidence for toxicity of nonfibrillar aggregates. Am J Pathol 159:1993-2000

34. Yoshioka A, Yamaya Y, Saiki S, Hirose G, Shimazaki K, Nakamura M, Ando Y (2001) A case of familial amyloid polyneuropathy homozygous for the transthyretin Val30Met gene with motor-dominant sensorimotor polyneuropathy and unusual sural nerve pathological findings. Arch Neurol 58:1914-1918

35. Hofer PA, Anderson R (1975) Postmortem findings in primary familial amyloidosis with polyneuropathy. Acta Pathol Microbiol Scand 83:309-322

36. Ikeda S, Yanagisawa N, Hongo M, Ito N (1987) Vagus nerve and celiac ganglion lesions in generalized amyloidosis. a correlative study of familial amyloid polyneuropathy and AL-amyloidosis. J Neurol Sci 79:129-139

37. Sobue G, Nakao N, Murakami K, Yasuda T, Sahashi K, Mitsuma T, Sasaki H (1990) Type I familial amyloid polyneuropathy. a pathological study of the peripheral nervous system. Brain 113:903-919

38. Hall AK, Landis SC (1992) Division and migration of satellite glia in the embryonic rat superior cervical ganglion. J Neurocytol 21:635-647

39. Hanani M (2005) Satellite glial cells in sensory ganglia: from form to function. Brain Res Brain Res Rev 48:457-476

40. Murakami T, Ohsawa Y, Sunada Y (2008) The transthyretin gene is expressed in human and rodend dorsal root ganglia. Neurosci Lett 436:335-339

41. Schlaepfer WW (1969) Experimental lead neuropathy: a disease of the supporting cells in the peripheral nervous system. J Neuropathol Exp Neurol 28:401-418

42. Kumamoto T, Fukuhara N, Miyatake T, Araki K, Takahashi Y, Araki S (1986) Experimental neuropathy induced by methyl mercury compounds: autoradiographic study of GABA uptake by dorsal root ganglia. Eur Neurol 25:269-277

43. Paresce DM, Ghosh RN, Maxfield FR (1996) Microglial cells internalize aggregates of the Alzheimer's disease amyloid beta-protein via a scavenger receptor. Neuron 17:553-565

44. Schionning JD, Danscher G (1999) Autometallographic mercurycorrelates with degenerative changes in dorsal root ganglia of rats intoxicated with organic mercury. APMIS 107:303-310

45. Pannese E, Procacci P (2002) Ultrastructural localization of NGF receptors in satellite cells of the rat spinal ganglia. J Neurocytol 31:755-763

46. Sorkin A, von Zastrow M (2009) Endocytosis and signalling: intertwining molecular networks. Nat Rev Mol Cell Biol 10:609-622

47. Jovic M, Sharma M, Rahajeng J, Caplan S (2010) The early endosome: a busy sorting station for proteins at the crossroads. Histol Histopathol 25:99-112

48. Mu FT, Callaghan JM, Steele-Mortimer O, Stenmark H, Parton RG, Campbell PL, McCluskey J, Yeo JP, Tock EP, Toh BH (1995) EEA1, an early endosome associated protein. EEA1 is a conserved alpha-helical peripheral membrane protein flanked by cysteine "fingers" and contains a calmodulin-binding IQ motif. J Biol Chem 270:13503-13511

49. Wyss-Coray T, Loike JD, Brionne TC, Lu E, Anankov R, Yan F, Silverstein SC, Husemann J (2003) Adult mouse astrocytes degrade amyloid-beta in vitro and in situ. Nat Med 9:453-457

50. Mohamed A, Posse de Chaves E (2011) A Internalization by neurons and glia. Int J Alzheimers Dis. doi: 10.4061/2011/127984

51. Suhr O, Danielsson A, Steen L (1992) Bile acid malabsorption caused by gastrointestinal motility dysfunction? an investigation of gastrointestinal disturbances in familial amyloidosis with polyneuropathy. Scand J Gastroenterol 27:201-207

52. Ando Y, Ohtsu Y, Terazaki H, Kibayashi K, Nakamura M, Ando E, Matsunaga N, Obayashi K, Uchino M, Ando M, Tsunenari S (2000) Japanese monozygotic twins with familial amyloidotic polyneuropathy (FAP) (ATTR Val30Met). Amyloid 7:133-136

53. Anan I, El-salhy M, Ando Y, Terazaki H, Suhr OB (2001) Comparison of amyloid deposits and infiltration of enteric nervous system in the upper with those in the lower gastrointestinal tract in patients with familial amyloidotic polyneuropathy. Acta Neuropathol 102:227-232

54. Guimarães A, Monteiro L, Coutinho P (1980) Pathology of the autonomic nervous system in andrade type of familial amyloidotic polyneuropathy. In: Glenner GG, Costa PP, de Freitas AF (eds) Amyloid and Amyloidosis. Excerpta Medica, Amsterdam, pp 88-98 
55. Anan I, El-Salhy M, Ando Y, Forsgren S, Nyhlin N, Terazaki H, Sakashita N, Suhr OB (1999) Colonic enteric nervous system in patients with familial amyloidotic neuropathy. Acta Neuropathol 98:48-54

56. Matsunaga N, Anan I, Forsgren S, Nagai R, Rosenberg P, Horiuchi S, Ando Y, Suhr OB (2002) Advanced glycation end products (AGE) and the receptor for AGE are present in gastrointestinal tract of familial amyloidotic polyneuropathy patients but do not induce NF-kappaB activation. Acta Neuropathol 104:441-447

57. Wixner J, Obayashi K, Ando Y, Karling P, Anan I (2013) Loss of gastric interstitial cells of Cajal in patients with hereditary transthyretin amyloidosis. Amyloid 20:99-106

58. Koike H, Kiuchi T, lijima M, Ueda M, Ando Y, Morozumi S, Tomita M, Kawagashira Y, Watanabe H, Katsuno M, Shimoyama Y, Okazaki Y, Kamei H, Sobue G (2011) Systemic but asymptomatic transthyretin amyloidosis 8 years after domino liver transplantation. J Neurol Neurosurg Psychiatry 82:1287-1290

\section{Submit your next manuscript to BioMed Central and take full advantage of:}

- Convenient online submission

- Thorough peer review

- No space constraints or color figure charges

- Immediate publication on acceptance

- Inclusion in PubMed, CAS, Scopus and Google Scholar

- Research which is freely available for redistribution 